\title{
Inserção profisssional e carreira de formandos e egressos brasileiros: revisão da literatura
}

\author{
Camila Gusmão de Almeida ${ }^{1}$ \\ Vera Socci \\ Universidade de Mogi das Cruzes, Mogi das Cruzes-SP, Brasil
}

\section{Resumo}

Considerando as características atuais do mundo do trabalho, juntamente com a ampla oferta de cursos de graduação, falar a respeito de desenvolvimento profissional e carreira é fundamental. O objetivo deste trabalho foi analisar a produção científica nacional de artigos disponíveis na SciELO, PePSIC, Redalyc e no Google Acadêmico, publicados no período que compreende os anos de 2010 a 2015, referentes à inserção profissional e carreira de formandos e egressos brasileiros. Foram selecionados 64 artigos, tendo sido analisados de maneira quantitativa e qualitativa, e constatou-se a homogeneidade de publicações no período pesquisado, com predominância de autoria múltipla e coautoria, maior quantidade de pesquisas quantitativas/qualitativas desenvolvidas em universidades públicas voltadas a egressos, em sua maioria na área das Ciências da Saúde.

Palavras-chave: desenvolvimento profissional, ensino superior, mercado de trabalho

\section{Abstract: Professional insertion and career of Brazilian undergraduates and graduates: literature review}

Considering the current characteristics of the work world, along with the wide range of graduation courses, there is a need to discuss about professional development and career. The objective of this study was to analyze the national scientific production of articles available in SciELO, PePSIC, Redalyc and Google Scholar, published during the period from 2010 to 2015, referring to the professional insertion and career of Brazilian undergraduates and graduates. A total of 64 articles were selected and analyzed in a quantitative and qualitative perspective. The homogeneity of publications in the studied period was verified, with a predominance of multiple authorship and co-authorship, a greater number of quantitative / qualitative research developed in public universities aimed at graduates, especially in the area of Health Sciences.

Keywords: professional development, higher education, labor market

\section{Resumen: Inserción profesional y carrera de estudiantes y egresados brasileños: revisión de literatura}

Considerando las características actuales del ámbito laboral y la amplia oferta de carreras universitarias, hablar sobre desarrollo profesional y de carrera es fundamental. El objetivo de este estudio fue analizar la producción científica nacional a partir de artículos disponibles en SciELO, PePSIC, Redalyc y Google Académico, publicados en el período de 2010 a 2015 y referentes a la inserción profesional y carrera de universitarios y egresados brasileños. Se seleccionaron 64 artículos, siendo analizados de manera cuantitativa y cualitativa. Se constató la homogeneidad de publicaciones en el período investigado, con predominio de autoría múltiple y coautoría, y mayor cantidad de investigaciones cuantitativas/cualitativas sobre egresados desarrolladas en universidades públicas, sobre todo en el área de las Ciencias de la Salud.

Palabras clave: desarrollo profesional, enseñanza superior, mercado laboral

\footnotetext{
${ }^{1}$ Endereço para correspondência: Rua Adelino Torquato, 120, Pq. Monte Líbano, Mogi das Cruzes-SP. E-mail: camilag.psi@hotmail.com
} 
Considerando o crescimento da produção científica em nosso país, que se deve a fatores como vinculação entre pesquisa acadêmica e instituições de Ensino Superior, exigência por produtividade e a busca por elevar o patamar do Brasil enquanto produtor de conhecimento (Kimura, 2010; Vilaça \& Palma, 2013; Waiczyk \& Ensslin, 2013), evidencia-se a importância da realização de uma análise para que seja possível visualizar o que vem sendo produzido em uma determinada área do conhecimento e identificar as lacunas existentes. A revisão sistemática de literatura viabiliza um agrupamento da literatura encontrada, sendo possível avaliá-la e sintetizar os resultados encontrados (Cordeiro, Oliveira, Rentería, \& Guimarães, 2007). O presente artigo tem como principal objetivo analisar publicações a respeito da inserção profissional e desenvolvimento de carreira de formandos e egressos brasileiros de cursos em nível superior, o que se deve especialmente a dois aspectos.

O primeiro aspecto seria a realidade contemporânea, considerando as mudanças ocorridas no mercado de trabalho, caracterizado atualmente por: globalização, evolução tecnológica, crises econômicas, fusões entre empresas, redução de funcionários e recursos, modificação de ocupações, flexibilidade e instabilidade (Bolles, 2000; Resende, 2003; Ribeiro, 2013), acarretando em modificações no modo de se construir as carreiras e trilhar o caminho profissional. Somam-se a isso o aumento das exigências feitas aos profissionais e a precarização do trabalho (Ribeiro, 2011). A instabilidade e mobilidade favorecem o aumento da insegurança dos trabalhadores, especialmente daqueles que estão entrando no mercado de trabalho, podendo isto contribuir para a dificuldade de inserção profissional dos jovens (Hannickel, 2004; Souza \& Castro, 2014).

$\mathrm{O}$ segundo aspecto revela-se pela importância de pesquisar sobre o momento de transição da universidade para o mercado de trabalho. De acordo com Basso (2008), ao longo da formação universitária, crises vocacionais podem ressurgir, pela necessidade de reafirmar a escolha profissional e também de se deparar efetivamente com a realidade ocupacional da profissão escolhida. Outros pontos destacados pela literatura que podem contribuir para dificuldades características deste momento seriam: falta de planejamento de carreira, dificuldades em atuar na área de formação, constatação do distanciamento entre o que foi aprendido na universidade e o que é esperado na prática profissional, desistência da busca pela satisfação profissional, realização de trabalhos alternativos e abandono do exercício profissional (Lucchiari, 1993; Mattosinho, Coelho, Meirelles, Souza, \& Argenta, 2010; Trintinália, 2011; Bardagi \& Hutz, 2012). Bardagi (2007) relata alta incidência de busca de serviços de orientação profissional por parte de graduandos e adultos jovens, ao enfrentarem dificuldades no desenvolvimento de carreira nesta fase da vida.

Há uma ênfase na realização de pesquisas voltadas à escolha profissional dos que ingressam no Ensino Superior e dos trabalhadores já inseridos no mercado de trabalho em comparação com trabalhos direcionados a graduandos e recém-formados. Também se priorizam pesquisas sobre a transição para o mercado de trabalho daqueles que não possuem Ensino Superior em comparação aos que possuem (Bardagi, 2007; Oliveira, Detomini, $\&$ Melo-Silva, 2013).

Para verificar a atenção dada a este momento de transição no que se refere à produção acadêmica e ao planejamento de ações que possam contribuir para mitigar dificuldades que possam acometer graduandos e recém-formados, inclusive no âmbito da Orientação Profissional e de Carreira, esta revisão de literatura tem como objetivo analisar a produção científica nacional de artigos indexados a respeito das temáticas desenvolvimento de carreira e inserção profissional de graduandos e egressos brasileiros.

\section{Método}

A busca pelos artigos científicos foi realizada em 2016, na biblioteca eletrônica SciELO (Scientific Electronic Library Online), no portal PePSIC (Periódicos Eletrônicos de Psicologia), no Google Acadêmico e no Sistema de Información Científica Redalyc, com a utilização dos descritores carreira, egressos, formandos e inserção profissional (no caso deste, foi utilizado o operador booleano "and", ou seja, "inserção and profissional").

Cada um dos sites foi consultado na sequência descrita, assim como os descritores refletem sua ordem de utilização. Quanto à busca dos artigos, procedeu-se da seguinte forma: a palavra-chave foi inserida combinando-a com o ano desejado, iniciando pelo ano de 2015 até o ano de 2010, de modo decrescente. Não houve restrição quanto ao idioma, considerando artigos em português, inglês ou espanhol.

Os critérios para inclusão no estudo foram: terem sido publicados entre os anos de 2010 a 2015, que se relacionassem diretamente a formandos ou egressos brasileiros, ou ainda estudos teóricos que se aproximassem do tema da pesquisa. Para estabelecer o que deveria ser considerado enquanto conceito de formando e egresso, a literatura foi consultada.

Nos artigos referentes aos formandos, considerou-se os estudantes que estivessem no penúltimo e último ano da graduação (Miano \& Vieira, 2012). Neste período de transição, reafirma-se a escolha profissional feita no 
momento de ingresso na graduação, e ocorre a procura por um emprego para aqueles que buscarão a inserção profissional a partir da profissão escolhida (Magalhães \& Teixeira, 2013; Silva \& Teixeira, 2013).

Buscando observar o momento de transição da universidade para o mercado de trabalho e o estabelecimento inicial da identidade profissional, foram selecionados estudos referentes a egressos com até seis anos de graduados, critério temporal estabelecido a partir das pesquisas de Gonçalves (2009), Souza (2009), Uvaldo (2010) e Teixeira et al. (2013).

A seleção dos artigos ocorreu em duas etapas: no momento de busca nos sites com a utilização dos descritores, foi feita uma seleção prévia dos artigos a partir da leitura dos títulos e resumos. A partir disto, foi feita uma segunda leitura mais aprofundada e seleção minuciosa, o que resultou na inclusão de 64 artigos científicos.

\section{Resultados e Discussão}

Após a seleção dos artigos, elaborou-se uma ficha para organizar os dados referentes a cada artigo, considerando os elementos: ano de publicação, autoria (se múltipla, coautoria ou única), instituição de origem (pública, privada, confessional ou mista), tipo de estudo (pesquisa ou artigo teórico), método (quantitativo/qualitativo ou somente qualitativo), caracterização dos participantes (formandos ou egressos), o(s) instrumento(s) utilizado(s) para coleta de dados no caso das pesquisas, os cursos pesquisados e suas áreas de conhecimento (estabelecidas conforme disposto na Plataforma Sucupira, disponível no site da CAPES) e as temáticas abordadas nos artigos.

Para as análises quantitativa e qualitativa, foram lidos os resumos dos artigos, ou os artigos na íntegra nos casos em que os resumos não ofereciam todas as informações necessárias. Quanto aos artigos que se originaram de dissertações e teses, estas foram consultadas para complementar o levantamento de informações quando necessário. Além disso, foi feito contato com os autores de alguns artigos para confirmar informações e esclarecer dúvidas quando isto se mostrou relevante. Para a análise estatística descritiva e não paramétrica, utilizou-se o programa BioEstat 5.0, com a realização do teste do qui-quadrado.

A escolha dos artigos mencionados no corpo do texto se deu a partir da consulta da ficha organizadora e dos resumos dos artigos. Buscou-se selecionar no mínimo um artigo para cada elemento analisado, considerando o modo como poderiam contribuir para a discussão frente aos resultados obtidos através da análise estatística.

Quanto ao ano de publicação, a maior frequência de publicações ocorreu em 2015, representando 20,31\% da amostra. Não houve diferença estatisticamente significante entre os anos de publicação $\left(\chi_{0}^{2}=1,06 ; \chi_{c}^{2}=11,07\right.$; $\mathrm{p}=0,9574 ; \mathrm{n}$. sig. $=0,05$ e n.g.l. $=5$ ), ou seja, manteve-se o interesse na realização de pesquisas voltadas às temáticas de inserção profissional e carreira de formandos e egressos.

Como exemplo de artigo publicado no ano de 2015, há o estudo de Furtado e Santiago, referente a egressos de Licenciatura em Educação Física da Universidade Federal de Goiás. Através da aplicação de questionários, observou-se que a docência e a atuação em academias se mostraram como os principais campos de atuação dos participantes da pesquisa. Grande parte dos egressos ingressaram na graduação com a intenção de atuarem em áreas relacionadas à saúde ou ao treinamento esportivo, no entanto, este era um curso de Licenciatura, cujo principal objetivo era formar profissionais para a educação básica. Além da ausência de cursos de Bacharelado em Educação Física no âmbito público no estado de Goiás até o ano de 2009, posterior ao ingresso dos participantes da pesquisa na graduação, a falta de informação a respeito dos tipos de cursos superior por parte dos vestibulandos revela que há maior necessidade de esclarecimento a respeito dos cursos de graduação e suas diferentes modalidades.

A autoria múltipla e a coautoria predominaram em relação à autoria única, apresentando porcentagem de $46,87 \%$ e $45,31 \%$ respectivamente, tendo a diferença sido estatisticamente significante $\left(\chi_{0}^{2}=18,78 ; \chi_{\mathrm{c}}^{2}=5,99\right.$; $\mathrm{p}=<0,0001 ;$ n.sig. $=0,05 ;$ n.g.l. $=2)$. A predominância da autoria múltipla e da coautoria é desejável, esperando-se que exista a colaboração entre centros de pesquisa.

Um exemplo de trabalho realizado por múltiplos autores é o de Lemos, Dubeux e Rocha-Pinto (2014), que verificou o impacto da graduação em Administração para a inserção profissional de formandos de uma universidade particular. Fatores como perfil socioeconômico, escolaridade dos pais e ocupação dos formandos foram comparados, tendo sido feita uma divisão entre os formandos com origem socioeconômica mais e menos favorável. Constatou-se que as diferenças socioeconômicas dos estudantes não tiveram impacto para sua inserção profissional, destacando a importância da formação enquanto fator que permitiu a entrada no mercado de trabalho e a minimização das desigualdades sociais.

O artigo de Dias e Soares (2012), desenvolvido em coautoria, analisou a escolha profissional de formandos relacionando isto com seu direcionamento de carreira. Os formandos foram entrevistados, e os resultados demonstraram que os entrevistados buscam um emprego que ofereça boa remuneração e estabilidade, havendo menor ênfase na escolha da atividade que irão exercer. Sentimentos 
como insegurança, indecisão e indiferença se apresentaram frente ao futuro profissional. As autoras enfatizaram a importância da realização de atividades de orientação para os formandos nas universidades, visando oferecer suporte para aqueles que irão ingressar no mercado de trabalho.

Houve uma predominância de publicações realizadas por universidades públicas $(57,81 \%)$ em relação às instituições privadas e confessionais $\left(\chi_{0}^{2}=68,34 ; \chi_{c}^{2}=9,49\right.$; $\mathrm{p}=<0,0001 ;$ n.sig. $=0,05 ;$ n.g.l. = 4). Esta diferença já é esperada, uma vez que nestas instituições há um incentivo mais expressivo à realização de pesquisas. $\mathrm{O}$ artigo de Trintinália, Riesco e Silva (2014) foi desenvolvido por uma instituição pública, no caso, a Universidade de São Paulo, e buscou conhecer a experiência universitária e a inserção profissional de egressos da primeira turma do curso de Obstetrícia da Escola de Artes, Ciências e Humanidades da USP. Entre os 24 entrevistados, 14 estavam inseridos no mercado de trabalho porém apenas 4 atuavam em sua área de formação. Esta graduação buscou atender à demanda por formação de obstetrizes, considerando as medidas do Ministério da Saúde para aumentar o número de realização de partos normais e humanizar as práticas na Obstetrícia. Foi ressaltado pelos participantes que com relação ao ingresso no mercado de trabalho, havia dificuldades por conta da falta de conhecimento a respeito desta profissão, além de divergências com o Conselho Regional de Enfermagem de São Paulo (Coren-SP). Muitos dos egressos buscaram alternativas como a atuação em outras atividades profissionais, o ingresso na pós-graduação e a mudança de país.

Em alguns casos, houve a parceria entre instituições públicas e privadas, e também entre instituições confessionais e privadas, como no artigo de Canever et al. (2014), que abordou a percepção de egressos de Enfermagem quanto à contribuição da graduação para sua inserção profissional, tendo sido elaborado a partir da colaboração entre uma universidade pública (Universidade Federal de Santa Catarina - UFSC) e uma universidade privada (Universidade do Extremo Sul de Santa Catarina - UNESC).

Tabela 1

Tipo de Estudo e Método

\begin{tabular}{lccc}
\hline & & $\mathrm{n}$ & $\%$ \\
\hline \multirow{2}{*}{ Tipo de estudo } & Pesquisa & 59 & 92,18 \\
& Teórico & 5 & 7,81 \\
\cline { 2 - 4 } & Total & 64 & 100 \\
\hline \multirow{3}{*}{ Método } & Quantitativo/qualitativo & 47 & 79,66 \\
& Qualitativo & 12 & 20,33 \\
\cline { 2 - 4 } & Total & 59 & 100 \\
\hline
\end{tabular}

Quanto ao tipo de estudo realizado, predominou a realização de pesquisas empíricas $(92,18 \%)$ em comparação aos estudos teóricos $\left(\chi_{0}^{2}=45,56 ; \chi_{c}^{2}=3,84 ; p=<0,0001\right.$; n.sig. $=0,05 ;$ n.g.l. $=1)$. Neste caso, o programa BioEstat 5.0 sugeriu a aplicação da correção de Yates, no qual o $\chi_{0}^{2}=43,89 ; p=<0,0001$.

É importante salientar que quando uma área do conhecimento está mais desenvolvida, há predominância de pesquisas empíricas em relação aos estudos teóricos, pois estas contribuem para o progresso e atualização do conhecimento, e com a publicação de artigos em periódicos, torna-se possível a divulgação dos resultados de maneira veloz (Oliveira \& Ferreira, 2007). Isto indica o desenvolvimento e o interesse por pesquisas que consideram o momento de transição universidade-mercado de trabalho.

Como exemplo de artigo teórico, o trabalho de Valore e Selig (2010) destacou as incertezas que marcam os tempos atuais, a falta de opção que muitas vezes se apresenta para os jovens no início de sua atuação profissional, a responsabilidade individual que é atribuída a cada um frente a seu próprio sucesso ou fracasso profissional e a necessidade de atualização e especialização constantes. Isto enfatiza a importância da reflexão a respeito dos temas carreira, inserção profissional e mercado de trabalho ao longo da formação, sendo necessário oferecer práticas de orientação e planejamento de carreira aos formandos.

Considerando a predominância da realização de pesquisas, foi feito o estudo a respeito do método utilizado, constatando que os estudos quantitativos/qualitativos foram maioria $(\chi 20=20,76 ; \chi 2 c=3,84 ; \quad p=0,0001 ;$ n.sig. $=0,05$; n.g.l. $=1)$, correção de Yates $(\chi 20=19,59 ; p=0,0001)$. Isto demonstra o desenvolvimento da área e a preocupação com a possibilidade de que sejam feitas generalizações fidedignas a partir dos resultados das pesquisas. Almeida, Rodrigues, 
Buriti e Witter (2007) ressaltam a importância da escolha do método conforme o objetivo estabelecido, pontuando que em casos de pesquisas quantitativas/qualitativas, o pesquisador, além de coletar os dados, também participa, compreende e interpreta as informações, sendo possível trabalhar com dados numéricos e verbais, ou seja, o fenômeno estudado pode ser abordado de maneiras diversas.

O artigo de Macedo e Batista (2011) abordou o preparo oferecido pela graduação em Medicina com relação aos aspectos trabalhistas e econômicos. Foi constatado que o modo como estes temas foram tratados durante a graduação foi insuficiente, revelando-se a necessidade de oferecer melhor suporte aos estudantes para facilitar a transição universidade-início da vida profissional. Conforme os autores, o enfoque quanti-qualitativo foi escolhido pela complementaridade dos métodos, contribuindo para oferecer uma perspectiva ampliada do contexto estudado.

Tabela 2

Participantes

\begin{tabular}{lcc}
\hline \multicolumn{1}{c}{ Participantes } & $\mathrm{n}$ & $\%$ \\
\hline Egressos (até 6 anos de formação) & 38 & 59,37 \\
Formandos & 21 & 32,81 \\
Não se aplica & 5 & 7,81 \\
Total & 64 & 100 \\
\hline
\end{tabular}

Quanto aos participantes das pesquisas descritas nos artigos, encontrou-se que os egressos representaram $59,37 \%$ do total e os formandos $32,81 \%$, havendo uma predominância de pesquisas voltadas a egres$\operatorname{sos}\left(\chi_{0}^{2}=25,53 ; \chi_{c}^{2}=5,99 ; \mathrm{p}=<0,0001 ;\right.$ n.sig. $=0,05$; n.g.l. =2). Isto pode ser explicado pela preocupação que há com as pessoas neste momento de transição e também pela avaliação dos cursos superiores realizadas pelo Instituto Nacional de Pesquisas Educacionais (INEP), pelo Ministério da Educação e Cultura (MEC) e também pela CAPES, o que acaba estimulando uma maior atenção a este período (Muritiba, Muritiba, Moura, \& Albuquerque, 2012; Barros \& Oliveira, 2013; Canever et al., 2014).
Silva e Teixeira (2013) comentam a diferença de identidades assumidas nas fases da graduação. No início da graduação, muitos estudantes estão na transição do final da adolescência para o começo da vida adulta e o papel a ser exercido será exclusivamente de estudante. Com o decorrer do curso, ocorre uma maior proximidade do papel de trabalhador, surgindo preocupações com a carreira profissional. Já no término da graduação, há uma transição mais definida de identidade, ocorrendo uma desvinculação do papel de estudante para a assunção do papel de profissional e construção da identidade profissional.

Tabela 3

Instrumentos Utilizados

\begin{tabular}{lccc}
\hline & Instrumento & $\mathrm{n}$ & $\%$ \\
\hline Questionário & 41 & 41,41 \\
Dados demográficos & 24 & 24,24 \\
Entrevista & 17 & 17,17 \\
Escalas & 7 & 7,07 \\
Não se aplica & 5 & 5,05 \\
Outros & 3 & 3,03 \\
Inventário & 2 & 2,02 \\
Total & 99 & 100 \\
\hline
\end{tabular}

Quanto aos instrumentos utilizados, sua importância se revela pela maneira como sua escolha possibilita que os dados sejam colhidos conforme o objetivo da pesquisa e o método. Na maioria dos artigos analisados, foi aplicado mais de um instrumento, em especial nas pesquisas qualitativas e quantitativas, o que se justifica 
pela necessidade de coleta de dados de diferentes maneiras. Houve grande expressividade da utilização de questionários $(41,41 \%)$, sendo sua predominância estatisticamente significante $\left(\chi_{0}^{2}=87,17 ; \chi_{\mathrm{c}}^{2}=12,59\right.$; $\mathrm{p}=<0,0001 ;$ n.sig. $=0,05$; n.g.l. = 6). O item "não se aplica" $(5,05 \%)$ refere-se aos estudos teóricos e o item "outros" $(3,03 \%)$ foi elaborado para englobar instrumentos que apareceram somente uma vez (grupo focal, questões abertas e Dados ENADE).

No artigo de Macedo e Batista (2011), cujo método era quantitativo/qualitativo, foram utilizados dois instrumentos de maneira complementar: um questionário aplicado a 26 residentes de Medicina e uma entrevista com 10 residentes. O questionário, previamente testado, dividia-se em três partes: caracterização do perfil pessoal e acadêmico dos participantes; questões fechadas do tipo Likert relativas ao ensino médico, planejamento de carreira, ética profissional e inserção no mercado de trabalho; e questões fechadas (certo-errado) referentes a conhecimentos sobre o mundo do trabalho (aspectos trabalhistas, previdenciários, tributários e inserção no mercado de trabalho). As entrevistas, com roteiro previamente testado, basearam-se nos seguintes tópicos: preparação para o mundo do trabalho durante a graduação médica; concepções acerca do mundo do trabalho e planejamento e expectativas em relação ao mundo do trabalho.

Quanto aos trabalhos que mencionaram o nome dos instrumentos utilizados, constatou-se que em sua maioria, tratavam-se de pesquisas conduzidas por psicólogos. Em dois destes trabalhos, foram feitas adaptações de instrumentos estrangeiros para o público brasileiro (Santos, Mognon, \& Joly, 2011; Magalhães \& Teixeira, 2013).

Mognon e Santos (2014) ressaltaram a escassez de instrumentos específicos para avaliar a experiência do estudante universitário. As autoras utilizaram a Escala de Avaliação da Vida Acadêmica (EAVA), cuja construção e validação encontra-se no artigo da autoria de Vendramini et al. (2004), e a Escala de Exploração Vocacional (EEV) (Teixeira, Bardagi, \& Hutz, 2007). Esta segunda escala (EEV) também foi utilizada na pesquisa de Bardagi e Boff (2010), além das Escalas de autoconceito e auto-eficácia profissional, presentes na tese de Teixeira (2002).

A utilização de teste com avaliação favorável do Conselho Federal de Psicologia (CFP), conforme lista disponível no site SATEPSI, ocorreu somente no artigo da autoria de Glaser e Bardagi (2011), no qual foram utilizados os seguintes instrumentos: questionário sociodemográfico e acadêmico; Inventário de Habilidades Sociais (IHS), da autoria de Del Prette e Del Prette (2001), que consta na lista de testes com avaliação favorável do CFP; e as Escalas de Auto-eficácia profissional e Decisão de Carreira, de Teixeira e Gomes (2005).

Portanto, entre os trabalhos analisados que apresentaram o nome dos instrumentos, observou-se que com apenas uma exceção, estes não foram submetidos à avaliação do CFP, porém, estavam indicados a autoria dos instrumentos utilizados e os estudos relativos a estes. Na opinião de Silva, Foch, Guimarães e Enumo (2014), muitos dos instrumentos não avaliados pelo SATEPSI podem possuir adequada capacidade psicométrica, sendo aplicados para fins de pesquisa.

Tabela 4

Áreas de Conhecimento dos Cursos Pesquisados

\begin{tabular}{lcc}
\hline \multicolumn{1}{c}{ Área de Conhecimento } & $\mathrm{n}$ & $\%$ \\
\hline Ciências da Saúde & 21 & 32,81 \\
Ciências Sociais Aplicadas & 16 & 25,00 \\
Misto & 11 & 17,18 \\
Engenharias & 4 & 6,25 \\
Não se aplica & 4 & 6,25 \\
Não consta & 3 & 4,68 \\
Ciências Exatas e da Terra & 2 & 3,12 \\
Linguística, Letras e Artes & 1 & 1,56 \\
Ciências Agrárias & 1 & 1,56 \\
Ciências Humanas & 1 & 1,56 \\
Total & 64 & 100 \\
\hline
\end{tabular}

Ao se analisar os cursos nos quais foram desenvolvidas as pesquisas, observou-se a realização de trabalhos em cursos mistos (em mais de um curso simultaneamente), representando $21,87 \%$ dos artigos. Foram desenvolvidos também trabalhos nos seguintes cursos: Administração (17,18\%); Enfermagem (12,50\%); Odontologia (4,68\%); 
Educação Física, Engenharia Civil, Fisioterapia, Matemática e Medicina (3,12\% cada); Biblioteconomia, Ciências Contábeis, Engenharia Florestal, Engenharia de Produção, Farmácia, Fonoaudiologia, Letras, Nutrição, Obstetrícia, Pedagogia e Turismo (1,56\% cada). O "não se aplica" $(6,25 \%)$ refere-se aos trabalhos que não trataram especificamente de nenhum curso, o que ocorreu nos trabalhos teóricos, com exceção do artigo de Takemoto, Werlang e Zeni (2015), que abordou o curso de Odontologia. Em três artigos analisados $(4,68 \%)$, não houve menção dos cursos nos quais a pesquisa foi realizada.

Quanto às áreas de conhecimento dos cursos pesquisados, notou-se a predominância de artigos relativos às Ciências da Saúde $(32,81 \%)\left(\chi_{0}^{2}=71,31 ; \chi_{\mathrm{c}}^{2}=16,92\right.$; $\mathrm{p}=<0,0001 ;$ n.sig. $=0,05$; n.g.l. $=9)$. Alguns elementos apareceram de maneira comum em artigos da área de Saúde, como a menção ao Sistema Único de Saúde (SUS), ao Programa de Saúde da Família (PSF) e também a mudança curricular de cursos (Böckmann, Motta, Camargo, Petry, \& Toassi, 2014; Canever et al., 2014).

A criação do SUS e do Programa de Saúde da Família foram medidas para ampliar e reformular a saúde pública brasileira, o que tornou necessária a formação de médicos generalistas, proporcionando o aumento dos postos de trabalho para profissionais da saúde (Pinheiro et al., 2011; Colenci \& Berti, 2012; Magalhães et al., 2012). As transformações que afetam o mercado de trabalho apontaram a necessidade de modificação do perfil destes profissionais, o que gerou reformulações em diversos cursos da área de Saúde (Santos, Holanda, Carolla, \& Traldi, 2014), indicadas em artigos da Enfermagem (Martinéli et al., 2011; Colenci \& Berti, 2012; Jesus, Gomes, Spillere, Prado, \& Canever, 2013; Arnold \& Rodrigues, 2014; Oliveira et al., 2014) e Odontologia (Böckmann et al., 2014).

Tabela 5

Temáticas Abordadas nos Artigos

\begin{tabular}{lcc}
\hline \multicolumn{1}{c}{ Temática } & $\mathrm{n}$ & $\%$ \\
\hline Trajetória profissional de egressos & 19 & 29,68 \\
Inserção profissional & 15 & 23,43 \\
Formação profissional & 12 & 18,75 \\
Direcionamento de carreira de formandos & 9 & 14,06 \\
Transição universidade-mercado de trabalho & 6 & 9,37 \\
Expectativas sobre a profissão & 1 & 1,56 \\
Exploração vocacional de formandos & 1 & 1,56 \\
Perfil de formandos & 1 & 1,56 \\
Total & 64 & 100 \\
\hline
\end{tabular}

Por fim, foi feita uma organização das temáticas abordadas nos artigos, considerando o tema central em cada artigo. As temáticas foram divididas em: Trajetória profissional de egressos, Inserção profissional, Formação profissional, Direcionamento de carreira de formandos, Transição universidade-mercado de trabalho, Perfil de Formandos, Exploração Vocacional de Formandos e Expectativas sobre a profissão.

Constatou-se a predominância de artigos que se referiam à Trajetória profissional de egressos $\left(\chi_{0}^{2}=42,25\right.$; $\chi_{\mathrm{c}}^{2}=14,07 ; \mathrm{p}=<0,0001 ;$ n.sig. $=0,05 ;$ n.g.l. $=7$ ). Esta categoria tratou do desenvolvimento de carreira daqueles que haviam terminado o curso universitário, considerando os seis primeiros anos após a graduação.

Exemplos de trabalhos que abordaram a temática Trajetória profissional de egressos foram os de Morais, Blanc, Domingues e Rosa (2010) e Bertinetti e Loureiro (2015), referentes ao desenvolvimento de carreira de egressos do curso de Administração de faculdades distintas. Nos trabalhos, nota-se a preocupação com o acompanhamento dos egressos por parte das instituições de formação, elemento que possivelmente se conecta com a solicitação do MEC quanto à avaliação dos cursos superiores e com a importância da verificação do percurso profissional dos egressos para a melhoria da qualidade dos cursos de graduação (Muritiba et al., 2012). Em ambos os trabalhos, foi feita uma caracterização do perfil dos egressos e análise da satisfação com o curso/profissão. A maioria dos participantes atuava em sua área de formação e afirmou estar satisfeita, destacando a contribuição da graduação para a melhoria de oportunidades na carreira.

Entre os artigos que trataram do tema Inserção profissional, enfatizando o momento de entrada no mercado de trabalho, há o trabalho de Salles, Farias e Nascimento (2015), que verificou como se encontravam os egressos do curso de Educação Física da Universidade 
Federal de Santa Catarina com relação à inserção profissional e perspectivas de continuidade da formação. Constatou-se que a maior parte dos egressos estava atuando em sua área de formação, principalmente em instituições privadas. Quanto à inserção profissional, foram considerados como fatores dificultadores a falta de experiência e a baixa remuneração, já como fatores favoráveis, destacaram-se as experiências e conhecimentos adquiridos ao longo da graduação e a vastidão do mercado de trabalho para este profissional.

Para o tema Formação profissional, referente aos artigos em que se discutia a qualidade da formação ou aspectos relacionados à graduação, há o trabalho de Gonçalves e Pereira (2015), cujo objetivo foi analisar a contribuição da graduação para a formação profissional e cidadã de egressos dos cursos de Medicina e Pedagogia da Universidade Estadual de Campinas. Enquanto formação cidadã, entende-se a importância do oferecimento de bases para o desenvolvimento da ética, pensamento reflexivo, senso crítico e responsabilidade social, somada aos conhecimentos inerentes às profissões. A partir da avaliação dos egressos de aspectos curriculares e vivências universitárias, constatou-se que a maior parte avalia positivamente sua formação e afirma aplicar os conhecimentos recebidos durante a graduação.

Quanto ao tema Direcionamento de carreira de formandos, que se refere à preparação dos formandos com relação à carreira e a atuação profissional, o artigo de Miano e Vieira (2012) abordou o comportamento de formandos de Administração frente à carreira, quanto a seu planejamento e gerenciamento. Entre os participantes, dois terços não fizeram nenhum tipo de planejamento de suas carreiras, e a maioria da amostra afirmou não ter conhecimento técnico de como gerir a própria carreira, apesar de cursarem uma graduação voltada ao mercado de trabalho. Também se observou que a existência de plano de carreira nas organizações em que os participantes trabalhavam ocorreu em casos isolados.

Para a temática Transição universidade-mercado de trabalho, Oliveira et al. (2013) descreveram as expectativas de formandos dos cursos de Psicologia e Administração quanto ao êxito que teriam na transição da universidade para o mercado de trabalho e os elementos que julgavam importantes para isto. Constatou-se que os formandos consideraram tanto características individuais como circunstâncias sociais, políticas e educacionais para o sucesso na transição. Como indicativos de sucesso, foram apontados: empregar-se na área de formação, aprovação em concurso público e a continuidade de formação por meio da realização de pós-graduação, alcançar a satisfação profissional, utilização das competências aprendidas no exercício profissional, ser influente e se tornar referência em sua profissão. Segundo os autores, há pouca preparação para a transição universidade-mercado de trabalho durante a graduação, sendo que esta preocupação passa a fazer parte do pensamento dos formandos apenas no último ano, indicando a importância de destinar mais atenção a isto.

Quanto ao tema Perfil de Formandos, o artigo de Böckmann et al. (2014) apresentou a análise do perfil de formandos do curso de Odontologia da Universidade Federal do Rio Grande do Sul, abrangendo aspectos sociodemográficos, motivos da escolha pelo curso, satisfação com a graduação, perspectivas de atuação profissional e continuidade da formação. Entre os participantes, houve a predominância de jovens, solteiros, sem filhos e mulheres, residentes do estado do Rio Grande do Sul. A maior parte dos formandos (90\%) declarou estar satisfeita com a escolha da profissão e com o curso, e a opção pela Odontologia se deu considerando fatores como satisfação pessoal e profissional, busca por posição social, estabilidade financeira, segurança e tranquilidade. A realização de especialização apareceu como predominante no que tange à intenção de continuidade aos estudos, e para a pretensão de atuação profissional, apareceram tanto a opção de atuação no serviço público como também privado.

Com relação à Exploração Vocacional de Formandos, que se refere à exploração realizada por universitários em busca de informações sobre si próprio e sobre o ambiente, sendo este conhecimento um facilitador para o desenvolvimento da carreira, o artigo de Mognon e Santos (2014) analisou o vínculo entre exploração vocacional e a vida acadêmica de formandos de cursos diversos de uma universidade paulista. Verificou-se que fatores como satisfação com o curso, certeza quanto à escolha e percepção das próprias habilidades influenciaram positivamente a exploração vocacional. Entre os participantes, as mulheres apresentaram maior crença em sua capacidade, maior busca por atividades extracurriculares e maior autoconhecimento. As autoras destacam a importância das experiências acadêmicas para o amadurecimento dos estudantes nos aspectos pessoal e profissional e sugerem que devem ser enfatizadas atividades como palestras, semanas de estudos, experiências práticas e estágios para aprimorar habilidades e fortalecer a crença de capacidade de conseguir arrumar emprego.

Por fim, para o tema Expectativas sobre a profissão, o artigo de Takemoto et al. (2015) abordou esta temática no âmbito da Odontologia. Através da realização de uma revisão de literatura, constatou-se que os estudantes de Odontologia possuem uma visão positiva da profissão, o que se apresenta em menor escala para os profissionais da área. Entre os estudantes, há uma tendência a uma visão 
mais idealizada da profissão no início do curso, considerando a atuação autônoma como uma boa opção, em comparação a seu término, na qual apresenta-se uma visão mais realista, em que o trabalho assalariado e o serviço público são avaliados como viáveis. Entre os profissionais, o serviço público também aparece como uma boa opção tanto para o aspecto de remuneração como também para a promoção da melhoria da saúde da população.

\section{Considerações Finais}

Esta revisão de literatura se propôs a analisar artigos nacionais que tratassem do momento transitório abrangendo o final da graduação até os anos iniciais do exercício profissional. Notou-se que no período pesquisado, houve interesse pelo desenvolvimento de pesquisas relacionadas à inserção profissional e desenvolvimento de carreira de formandos e egressos, sendo a maior parte delas realizadas em autoria múltipla e coautoria, em universidades públicas, em especial na área das Ciências da Saúde.

Considerando a amostra de artigos pesquisados, houve uma predominância de pesquisas direcionadas aos egressos, quando comparadas às pesquisas direcionadas aos formandos. Observa-se uma preocupação em investigar como tem se dado a entrada no mercado de trabalho após o término da graduação e também o percurso feito pelos egressos. Aspectos como estratégias utilizadas para ingressar no mercado, perfil dos egressos, avaliação da formação, intenção da continuidade dos estudos, locais de atuação, remuneração, entre outros, apareceram como temas de interesse dos pesquisadores.

Quanto à avaliação da formação, constatou-se que a maior parte das pesquisas foi desenvolvida com egressos, buscando conhecer a opinião desta população a respeito da formação. Sugere-se o desenvolvimento de pesquisas de avaliação da formação ao longo da graduação, inclusive através de acompanhamentos longitudinais, o que possibilitaria verificar os fatores considerados importantes bem como o que poderia ser melhorado para complementar a formação.

Já os artigos relativos à Transição da universidade para o mercado de trabalho se concentraram nos formandos, com apenas uma exceção. Um ponto a destacar é que entre todas as pesquisas, há somente uma menção ao suporte a formandos durante a graduação, através do oferecimento de uma disciplina optativa. Também se constatou a ausência de ações durante a formação ao se analisar os artigos relacionados ao Direcionamento de carreira e à Exploração vocacional de formandos. A partir disto, sugere-se a necessidade do desenvolvimento de ações e pesquisas que se voltem à importância da realização de medidas eficazes para favorecer a reflexão e o planejamento da carreira para graduandos, inclusive de maneira gradual ao longo da formação. Esta necessidade também foi destacada em algumas pesquisas (Bardagi \& Boff, 2010; Dias \& Soares, 2012; Kreutz, 2012).

Apesar das limitações do estudo, que optou por analisar somente artigos publicados a partir de um recorte temporal bastante delimitado, visando um momento de transição específico, espera-se que as discussões realizadas possam contribuir e chamar atenção para a importância do momento transitório que se dá frente à saída da universidade e entrada no conturbado mercado de trabalho atual. É necessário repensar a qualidade das formações oferecidas nas instituições de ensino superior de nosso país, que muitas vezes prepara os estudantes a partir de um referencial somente técnico, sem colaborar mais efetivamente para o desenvolvimento de competências importantes para que os estudantes possam se constituir como profissionais capazes para atuarem na sociedade.

Destaca-se a necessidade de considerar o desenvolvimento não só de pesquisas, mas de ações, inclusive no âmbito da área de Orientação Profissional e de Carreira, que busquem contribuir para o acompanhamento de formandos e egressos frente a este momento transitório de maneira mais difundida, conscientizando a população de que este é um momento que merece atenção tanto quanto o ingresso no Ensino Superior.

\section{Referências}

Almeida, I. A., Rodrigues, L. O., Buriti, M. A., \& Witter, G. P. (2007). Meta-análise da Produção Científica sobre Psicologia do Idoso no LILACS (1991-2003). In: M. A. Buriti, C. Witter, \& G. P. Witter (Orgs.), Produção Científica e Psicologia Educacional (pp. 57-78). Guararema: Anadarco.

Arnold, K. S., \& Rodrigues, R. M. (2014). Formação para a área de saúde coletiva em um curso de graduação em Enfermagem na visão dos egressos. FIEP Bulletin - Online, 84, Special Edition - Article II, s/p.

Bardagi, M. P. (2007). Evasão e comportamento vocacional de universitários: Estudos sobre o desenvolvimento de carreira na graduação. Tese de Doutorado não-publicada, Universidade Federal do Rio Grande do Sul, Porto Alegre.

Bardagi, M. P., \& Boff, R. M. (2010). Autoconceito, auto-eficácia profissional e comportamento exploratório em universitários concluintes. Avaliação: Revista da Avaliação da Educação Superior, 15, 41-56. DOI: https://dx.doi. org/10.1590/S1414-40772010000100003 
Bardagi, M. P., \& Hutz, C. S. (2012). Mercado de trabalho, desempenho acadêmico e o impacto sobre a satisfação universitária. Revista de Ciências Humanas, 46, 183-198. DOI: http://dx.doi.org/10.5007/2178-4582.2012v46n1p183

Barros, A. C. N., \& Oliveira, V. R. C. (2013). Mercado de Trabalho: Perspectivas de Concluintes de Cursos de Fisioterapia. Estudos - Vida e Saúde, 40, 507-526.

Basso, C. (2008). Escolha profissional: estudantes universitários em crise durante as fases intermediárias da formação acadêmica. Dissertação de mestrado não-publicada, Universidade Federal de Santa Catarina, Florianópolis.

Bertinetti, M. P., \& Loureiro, M. H. F. (2015). Colocação Profissional e Inserção no Mercado de Trabalho dos Alunos Egressos do Curso de Administração da Faculdade de Ciências Sociais de Guarantã do Norte - MT, entre os anos de 2011 a 2013. Nativa - Revista de Ciências Sociais do Norte do Mato Grosso, 4(1), 1-19.

Böckmann, F. S., Motta, B. B., Camargo, J. M., Petry, P. C., \& Toassi, R. F. C. (2014). The profile of Dentistry students at Federal University of Rio Grande do Sul and expectations regarding the profession, 2010-2011. RGO - Revista Gaúcha de Odontologia, 62, 267-274. DOI: https://dx.doi.org/10.1590/1981-8637201400030000062400

Bolles, R. N. (2000). Como conseguir um emprego e descobrir a sua profissão ideal: Qual a cor do seu pára-quedas? (2a ed.). Rio de Janeiro: Sextante.

Canever, B. P., Gomes D. C., Jesus, B. H., Spillere, L. B., Prado M. L., \& Backes, V. M. S. (2014). Processo de formação e inserção no mercado de trabalho: uma visão dos egressos de enfermagem. Revista Gaúcha de Enfermagem, 35(1), 87-93.

Colenci, R., \& Berti, H. W. (2012). Formação profissional e inserção no mercado de trabalho: percepções de egressos de graduação em enfermagem. Revista da Escola de Enfermagem da USP, 46, 158-166. DOI: https://dx.doi.org/10.1590/ S0080-62342012000100022

Cordeiro, A. M., Oliveira, G. M., Rentería, J. M., \& Guimarães, C. A. (2007). Revisão sistemática: uma revisão narrativa. Revista do Colégio Brasileiro de Cirurgiões, 34, 428-431. DOI: https://dx.doi.org/10.1590/S0100-69912007000600012

Del Prette, Z. A. P., \& Del Prette, A. (2001). Inventário de Habilidades Sociais - manual de aplicação, apuração e interpretação. São Paulo: Casa do Psicólogo.

Dias, M. S. L., \& Soares, D. H. P. (2012). A escolha profissional no direcionamento da carreira dos universitários. Psicologia: Ciência e Profissão, 32, 272-283. DOI: https://dx.doi.org/10.1590/S1414-98932012000200002

Furtado, R. P., \& Santiago, L. P. (2015). Educação Física e trabalho: considerações a respeito da inserção profissional de egressos da FEF-UFG. Revista Brasileira de Educação Física e Esporte, 29, 325-336. DOI: https://dx.doi. org/10.1590/1807-55092015000200325

Glaser, S. L., \& Bardagi, M. P. (2011). Habilidades sociais, auto-eficácia e decisão de carreira em universitários em no final de curso. Boletim Academia Paulista de Psicologia, 80, 148-165.

Gonçalves, J. A. (2009). Desenvolvimento profissional e carreira docente - Fases da carreira, currículo e supervisão. Sisifo: Revista de Ciências da Educação, 8, 23-36.

Gonçalves, M. L., \& Pereira, E. M. A. (2015). Contribuições da educação geral na formação de médicos e pedagogos egressos de uma universidade pública. Avaliação: Revista da Avaliação da Educação Superior (Campinas), 20, 513530. DOI: https://dx.doi.org/10.590/S1414-40772015000200012

Hannickel, J. T. (2004). E quando eu me formar?: leitura para universitários. Petrópolis: Vozes.

Jesus, B. H., Gomes, D. C., Spillere, L. B. B., Prado, M. L., \& Canever, B. P. (2013). Inserção no mercado de trabalho: trajetória de egressos de um curso de graduação em enfermagem. Escola Anna Nery, 17, 336-345. DOI: https:// dx.doi.org/10.1590/S1414-81452013000200019

Kimura, E. T. (2010). O dilema das revistas científicas brasileiras na divulgação da produção científica nacional. Arquivos Brasileiros de Endocrinologia \& Metabologia, 54, 1-2. DOI: https://dx.doi.org/10.1590/S0004-27302010000100001

Kreutz, D. H. (2012). A percepção dos formandos em Administração de empresas do Centro Universitário UNIVATES sobre planejamento e gestão de carreira. Revista Destaques Acadêmicos, 4(1), 7-28. DOI: http://dx.doi.org/10.22410/ issn.2176-3070.v4i1a2012.133

Lemos, A. H. C., Dubeux, V. J. C., \& Rocha-Pinto, S. R. (2014). Educação Superior, Inserção Profissional e Origem Social: Limites e Possibilidades. Sociedade, Contabilidade e Gestão, 9(1), 48-64.

Lucchiari, D. H. P. S. (1993). Pensando e vivendo a orientação profissional (5a ed). São Paulo: Summus.

Macedo, D. H., \& Batista, N. A. (2011). O Mundo do Trabalho durante a graduação médica: a visão dos recém-egressos. Revista Brasileira de Educação Médica, 35, 44-51.

Magalhães, A. P. S., Esteves, C. C., Elias, S. F., Oliveira, L. D., Figueredo, N. D. M., \& Costa, I. D. (2012). Perfil dos egressos de Medicina de uma Faculdade de Medicina de Juiz de Fora/MG. Revista Ciências em Saúde, 2(2), 32-44. DOI: http://dx.doi.org/10.21876/rcsfmit.v2i2.98 
Magalhães, M. O., \& Teixeira, M. A. P. (2013). Antecedentes de comportamentos de busca de emprego na transição da universidade para o mercado de trabalho. Psicologia: Teoria e Pesquisa, 29, 411-419.

Martinéli, D. D., Moura, C. R., Cesarino, C. B., Beccaria, L. M., Pinto, M. H., \& Paschoal, V. A. (2011). Avaliação do currículo da graduação em Enfermagem por egressos. Cogitare Enfermagem, 16, 524-529.

Mattosinho, M. M. S., Coelho, M. S., Meirelles, B. H. S., Souza, S. S., \& Argenta, C. E. (2010). Mundo do trabalho: alguns aspectos vivenciados pelos profissionais recém-formados em enfermagem. Acta Paulista de Enfermagem, 23, 466-471. DOI: https://dx.doi.org/10.1590/S0103-21002010000400004

Miano, V. Y., \& Vieira, F. O. (2012). Perspectivas de carreira dos formandos de Administração de uma IFES. Revista Pensamento Contemporâneo em Administração, 6(1), 72-90. DOI: http://dx.doi.org/10.12712/rpca.v6i1.114

Mognon, J. F., \& Santos, A. A. A. (2014). Vida acadêmica e exploração vocacional em universitários formandos: relações e diferenças. Estudos e Pesquisas em Psicologia, 14, 89-106.

Morais, A. G., Blanc, J., Domingues, L. J., \& Rosa, R. T. (2010). A inserção dos egressos do curso de Administração da faculdade União das Américas no mercado de trabalho. Pleiade, 4(8), 127-139.

Muritiba, P. M., Muritiba, S. N., Moura, M. J. S. B., \& Albuquerque, L. G. (2012). Satisfação dos egressos em Administração, Economia e Contabilidade e desempenho profissional. Revista Alcance - Eletrônica, 19, 308-326. DOI: http://dx.doi.org/10.14210/alcance.v19n3.p308-326

Oliveira, F. M. M., Vasconcelos, M. I. O., Vieira, I. P. G. F., Ferreira, A. G. N., Cavalcante, A. S. P., \& Teófilo, F. S. (2014). Inserção de egressos do curso de graduação em Enfermagem no mercado de trabalho. SANARE, 13(1), 92-98.

Oliveira, M. C., Detomini, V. C., Melo-Silva, L. L. (2013). Sucesso na transição universidade-trabalho: expectativas de universitários formandos. Psicologia em Revista, 19, 497-518. DOI: https://dx.doi.org/DOI - 10.5752/P.1678-9563.2013v19n3p497

Oliveira, P., \& Ferreira, A. A. (2007). Autismo: Análise da produção científica arrolada na PsycINFO (2004). In C. Witter, M. A. Buriti \& G. P. Witter (Orgs.), Problemas Psicossociais: análise de produção (pp. 129-146). Guararema: Anadarco.

Pinheiro, V. C., Menezes, L. M. B., Aguiar, A. S. W., Moura, W. V. B., Almeida, M. E. L., \& Pinheiro, F. M. C. (2011). Inserção dos egressos do curso de odontologia no mercado de trabalho. RGO-Revista Gaúcha de Odontologia, 59, 277-283.

Resende, E. (2003). O livro das competências: desenvolvimento das competências: a melhor auto-ajuda para pessoas, organizações e sociedade (2a ed.). Rio de Janeiro: Qualitymark.

Ribeiro, M. A. (2011). Juventude e trabalho: construindo a carreira em situação de vulnerabilidade. Arquivos Brasileiros de Psicologia, 63(número especial), 58-70.

Ribeiro, M. A. (2013). Sistematização das principais narrativas produzidas sobre carreira na literatura especializada. Revista Brasileira de Orientação Profissional, 14, 177-189.

Salles, W. N., Farias, G. O., \& Nascimento, J. V. (2015). Inserção profissional e formação continuada de egressos de cursos de graduação em Educação Física. Revista Brasileira de Educação Física e Esporte, 29, 475-486. DOI: https://dx.doi.org/10.1590/1807-55092015000300475

Santos, A. A. A., Mognon, J. F., \& Joly, M. C. R. A. (2011). Crenças de autoeficácia na transição para o trabalho em formandos de engenharia. Revista Brasileira de Orientação Profissional, 12, 197-204.

Santos, M. G., Holanda, F. L., Carolla, D. C., \& Traldi, M. C. (2014). Egressos do Curso de Enfermagem da Faculdade de Medicina de Jundiaí: perfil e inserção no mercado de trabalho. Perspectivas Médicas, 25(1), 33-42.

Silva, A. M. B., Foch, G. F. L., Guimarães, C. A., \& Enumo, S. R. F. (2014). Instrumentos aplicados em estudos brasileiros em psicologia do esporte. Estudos Interdisciplinares em Psicologia, 5(2), 77-95. DOI: http://dx.doi. org/10.5433/2236-6407.2014v5n2p77

Silva, C. S. C., \& Teixeira, M. A. P. (2013). Experiências de Estágio: Contribuições para a Transição UniversidadeTrabalho. Paidéia (Ribeirão Preto), 23, 103-112. DOI: https://dx.doi.org/10.1590/1982-43272354201312

Souza, D. B. (2009). Os dilemas do professor iniciante: reflexões sobre os cursos de formação inicial. Saber Acadêmico, 8 , p. 35-45.

Souza, M. G. C., \& Castro, L. R. (2014). O projeto profissional de jovens das classes médias: orientações normativas e estratégias de inserção. Cadernos de Psicologia Social do Trabalho, 17, 161-175. DOI: http://dx.doi.org/10.11606/ issn.1981-0490.v17i2p161-175

Takemoto, M., Werlang, F., \& Zeni, E. (2015). Expectativas de estudantes e profissionais sobre o curso de Odontologia. Revista Tecnológica, 2(1), 257-270. 
Teixeira, L. C., Rodrigues, A. L. V., Santos, J. N., Cardoso, A. F. R., Gama, A. C. C., \& Resende, L. M. (2013). Trajetória profissional de egressos em Fonoaudiologia. Revista CEFAC, 15, 1591-1600. DOI: https://dx.doi.org/10.1590/ S1516-18462013005000048

Teixeira, M. A. P. (2002). A experiência de transição entre a universidade e o mercado de trabalho na adultez jovem. Tese de Doutorado não-publicada, Universidade Federal do Rio Grande do Sul, Porto Alegre.

Teixeira, M. A. P., Bardagi, M. P., \& Hutz, C. S. (2007). Escalas de exploração vocacional (EEV) para universitários. Psicologia em Estudo, 12, 195-202. DOI: https://dx.doi.org/10.1590/S1413-73722007000100023

Teixeira, M. A. P., \& Gomes, W. B. (2005). Decisão de carreira entre estudantes em fim de curso universitário. Psicologia: Teoria e Pesquisa, 21, 327-334.

Trintinália, M. M. J. (2011). Caracterização e inserção profissional de egressos do curso de graduação em Obstetrícia da Universidade de São Paulo. Dissertação de mestrado não-publicada, Universidade de São Paulo, São Paulo.

Trintinália, M. M. J., Riesco, M. L. G., \& Silva, L. C. F. P. (2014). Caracterização e inserção profissional de egressos da graduação em Obstetrícia da Universidade de São Paulo. Revista Educação, 9(1), 11-23.

Uvaldo, M. C. C. (2010). Tecendo a trama identitária: um estudo sobre mudanças de carreira. Tese de doutorado nãopublicada, Universidade de São Paulo, São Paulo.

Valore, L. A., \& Selig, G. A. (2010). Inserção profissional de recém-graduados em tempos de inseguranças e incertezas. Estudos e Pesquisas em Psicologia, 10, 390-404.

Vendramini, C. M. M., Santos, A. A. A., Polydoro, S. A. J., Sbardelini, E. T. B., Serpa, M. N. F., \& Natário, E. G. (2004). Construção e validação de uma escala sobre avaliação da vida acadêmica (EAVA). Estudos de Psicologia (Natal), 9 , 259-268. DOI: https://dx.doi.org/10.1590/S1413-294X2004000200007

Vilaça, M. M., \& Palma, A. (2013). Diálogo sobre cientometria, mal-estar na academia e a polêmica do produtivismo. Revista Brasileira de Educação, 18, 467-484. DOI: https://dx.doi.org/10.1590/S1413-24782013000200013

Waiczyk, C., \& Ensslin, E. R. (2013). Avaliação de produção científica de pesquisadores: mapeamento das publicações científicas. Revista Contemporânea de Contabilidade, 10(20), 97-112. DOI: http://dx.doi. org/10.5007/2175-8069.2013v10n20p97

Recebido: 06/05/2017

$1^{a}$ reformulação: $25 / 07 / 2017$

Aceite final: 01/09/2017

Sobre as autoras

Camila Gusmão de Almeida é Psicóloga com Especialização em Psicologia Organizacional pela Universidade de Mogi das Cruzes.

Vera Socci é Professora Adjunta Aposentada pela Universidade Federal Fluminense. Professora Titular da Universidade de Mogi das Cruzes. Coordenadora do Mestrado Profissional em Psicogerontologia do Instituto Educatie de Ensino e Pesquisa de Mogi das Cruzes. 